\title{
Exacerbation of psoriasis with $\beta$-blocker therapy
}

\section{Salman Waqar, Pradip K Sarkar}

A 54-year-old man with a 20-year history of erythrodermic psoriasis underwent coronary artery bypass graft surgery. The patient had no prior history of $\beta$-blocker use. He was given the $\beta$-blocker bisoprolol postoperatively to treat atrial fibrillation. Within 72 hours after beginning therapy with bisoprolol, the patient experienced an acute worsening of the psoriasis. Generalized inflammation of the skin developed with deep, erythematous, coalescing plaques (Figure 1). The patient's face was not affected, which is characteristic of erythrodermic psoriasis.

The patient lost substantial transepidermal fluid from a large area of denuded skin. This fluid loss resulted in hypovolemia and hemodynamic instability that required aggressive fluid therapy. The psoriasis was treated aggressively with methotrexate and topical agents. The bisoprolol was discontinued because of concern that it may have caused the exacerbation of psoriasis. The patient's condition improved after discontinuation of the bisoprolol.

When managing atrial fibrillation, the initial goals are anticoagulation and control of the heart rate. The eventual goal is to restore and maintain sinus rhythm. ${ }^{1}$ B-blockers are the drugs of choice for controlling heart rate, ${ }^{2}$ but have been reported to cause severe worsening of psoriasis resulting in hemodynamic compromise. ${ }^{3}$ This reaction is extremely rare.

The mechanism for the exacerbation of psoriasis with $\beta$-blocker use is thought to be related to a blockade in the activation of the messenger system of cyclic adenosine 3',5'-cyclic monophosphate. This blockade results in reduced intracellular concentrations of calcium. This reduction may, in turn, cause an accelerated proliferation of keratinocytes or polymorphonuclear leukocytes, both of which may play a role in inducing or exacerbating psoriasis. ${ }^{4}$

From the Department of Cardiothoracic Surgery, Sheffield Teaching Hospitals NHS Foundation Trust, Sheffield, UK

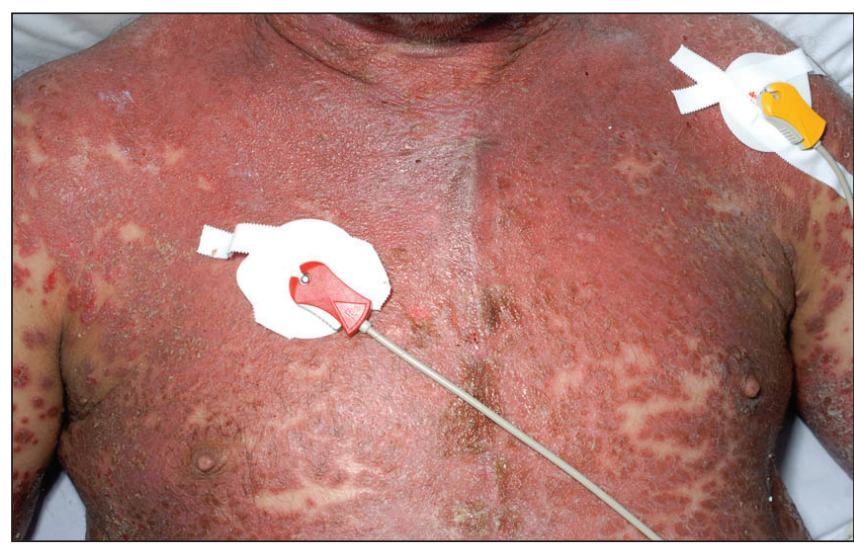

Figure 1: Photograph of the chest of a 54-year-old man with acute exacerbation of psoriasis during postoperative therapy with bisoprolol. The image shows generalized inflammation and coalescing plaques across the site of the sternotomy.

\section{REFERENCES}

1. Olshansky B, Rosenfeld LE, Warner AL, et al. The atrial fibrillation follow-up investigation of rhythm management (AFFIRM) study: approaches to control rate in atrial fibrillation. J Am Coll Cardiol 2004;43:1201-8

2. Hagens VE, Ranchor AV, Van Sonderen E, et al. Effect of rate or rhythm control on quality of life in persistent atrial fibrillation. Results from the rate control versus electrical cardioversion (RACE) study. J Am Coll Cardiol 2004;43:241-7.

3. Jha PK, Das SR, Musleh GS, et al. Psoriasis-induced postoperative cardiac failure. Ann Thorac Surg 2005;79:1390-1.

4. O'Brien M, Koo J. The mechanism of lithium and beta-blocking agents in inducing and exacerbating psoriasis. J Drugs Dermatol 2006;5:426-32.

Clinical images are chosen because they are particularly intriguing, classic or dramatic. Submissions of clear, appropriately labelled high-resolution images must be accompanied by a figure caption and the patient's written consent for publication. A brief explanation (300 words maximum) of the educational significance of the images with minimal references is required. 